\title{
Multiparty Computation for Modulo Reduction without Bit-Decomposition and a Generalization to Bit-Decomposition ${ }^{\star}$
}

\author{
Chao Ning and Qiuliang $\mathrm{Xu}^{\star \star}$ \\ School of Computer Science and Technology, Shandong University, \\ Jinan, 250101, China \\ ncnfl@mail.sdu.edu.cn, xql@sdu.edu.cn
}

\begin{abstract}
Bit-decomposition, which is proposed by Damgård et al., is a powerful tool for multi-party computation (MPC). Given a sharing of secret $a$, it allows the parties to compute the sharings of the bits of $a$ in constant rounds. With the help of bit-decomposition, constant-rounds protocols for various MPC problems can be constructed. However, bitdecomposition is relatively expensive, so constructing protocols for MPC problems without relying on bit-decomposition is a meaningful work. In multi-party computation, it remains an open problem whether the modulo reduction problem can be solved in constant rounds without bitdecomposition.

In this paper, we propose a protocol for (public) modulo reduction without relying on bit-decomposition. This protocol achieves constant round complexity and linear communication complexity. Moreover, we show a generalized bit-decomposition protocol which can, in constant rounds, convert the sharing of secret $a$ into the sharings of the digits of $a$, along with the sharings of the bits of every digit. The digits can be base- $m$ for any $m \geq 2$.
\end{abstract}

Keywords: Multiparty Computation, Constant-Rounds, Modulo Reduction, Generalization to Bit-Decomposition.

\section{Introduction}

Secure multi-party computation (MPC) allows the computation of a function $f$ when the inputs to $f$ are secret values held by distinct parties. After running the MPC protocol, the parties obtains only the predefined outputs but nothing else, and the privacy of their inputs are guaranteed. Although generic solutions for MPC already exist [210], the efficiency of these protocols tends to be low. So we focus on constructing efficient protocols for specific functions. More exactly, we are interested in integer arithmetic in the information theory setting [12].

\footnotetext{
* Supported by the National Natural Science Foundation of China under Grant No. 60873232.

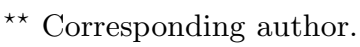


A proper choice of representation of the inputs can have great influence on the efficiency of the computation [7/18]. For example, when we want to compute the sum or the product of some private integer values, we'd better represent these integers as elements of a prime field $\mathbb{Z}_{p}$ and perform the computations using an arithmetic circuit as additions and multiplications are trivial operations in the field. If we use the binary representation of the integers and a Boolean circuit to compute the expected result, then we will get a highly inefficient protocol as the bitwise addition and multiplication are very expensive [45]. On the other hand, if we want to compare some (private) integer values, the binary representation will be of great advantage as comparison is a bit-oriented operation. In this case, the arithmetic circuit over $\mathbb{Z}_{p}$ will be a bad choice.

To bridge the gap between the arithmetic circuits and the Boolean circuits, Damgård et al. 7] proposed a novel protocol, called bit-decomposition, to convert a sharing of secret $a$ into the sharings of the bits of $a$. This protocol is very useful both in theory and application. However, the bit-decomposition protocol is relatively expensive in terms of round and communication complexities. So the work on constructing (constant-rounds) protocols for MPC problems without relying on bit-decomposition is not only interesting but also meaningful. Recently, in [12, Nishide et al. constructed more efficient protocols for comparison, interval test and equality test of shared secrets without relying on the bit-decomposition protocol. However, it remains an open problem whether the modulo reduction problem can be solved in constant rounds without bit-decomposition [17. In this paper, we show a linear protocol for the (public) modulo reduction problem without relying on bit-decomposition. What's more, the bit-decomposition protocol of [7] can only de-composite the sharing of secret $a$ into the sharings of the bits of $a$. However, especially in practice, we may often need the sharings of the digits of $a$. Here the digits can be base- $m$ for any $m \geq 2$. For example, in real life, integers are (almost always) represented as base-10 digits. Then, MPC protocols for practical use may often require the base-10 digits of the secret shared integers. Another example is as follows. If the integers are about time and date, then base-24, base-30, base-60, or base-365 digits may be required. So, to meet these requirements, we propose a generalization to bit-decomposition in this paper.

\subsection{Our Contributions}

First we introduce some necessary notations. We focus mainly on the multi-party computation based on linear secret sharing schemes. Assume that the underlying secret sharing scheme is built in field $\mathbb{Z}_{p}$ where $p$ is a prime with bit-length $l$ (i.e. $l=\lceil\log p\rceil)$. For secret $a \in \mathbb{Z}_{p}$, we use $[a]_{p}$ to denote the secret sharing of $a$, and $[a]_{B}$ to denote the sharings of the bits of $a$, i.e. $[a]_{B}=\left(\left[a_{l-1}\right]_{p}, \ldots,\left[a_{1}\right]_{p},\left[a_{0}\right]_{p}\right)$.

The public modulo reduction problem can be formalized as follows:

$[x \bmod m]_{p} \leftarrow$ Modulo-Reduction $\left([x]_{p}, m\right)$

where $x \in \mathbb{Z}_{p}$ and $m \in\{2,3, \ldots, p-1\}$. 
In existing public modulo reduction protocols [7]17, the bit-decomposition is involved, incurring $O(l \log l)$ communication complexity. What's more, in the worst case, the communication complexity of this protocol may go up to $\Theta\left(l^{2}\right)$. Specifically, the existing modulo reduction protocol uses the bit-decomposition protocol to reduce the "size" of the problem, and then uses up to $l$ comparisons, which is non-trivial, to determine the final result. This is essentially an "exhaustive search". If the bit-length of the inputs to the comparison protocol is relatively long, e.g. $\Theta(l)$ which is often the case, the overall complexity will go up to $\Theta\left(l^{2}\right)$. So, the efficiency of the protocol may be very poor. To solve this problem, we propose a protocol, which achieves constant round complexity and linear communication complexity, for public modulo reduction without relying on bit-decomposition. Besides this, we also propose an enhanced protocol that can output the sharings of the bits of $x \bmod m$, i.e. $[x \bmod m]_{B}$.

Moreover, we also construct a generalized bit-decomposition protocol which can, in constant rounds, convert the sharing of secret $a$ into the sharings of the digits of $a$, along with the sharings of the bits of every digit. The digits can be base- $m$ for any $m \geq 2$. We name this protocol the Base-m Digit-BitDecomposition Protocol. The asymptotic communication complexity of this protocol is $O(l \log l)$. Obviously, when $m$ is a power of 2, this protocol degenerates to the bit-decomposition protocol.

For illustration, we will show an example here. Pick a binary number

$$
a=(11111001)_{2}=249 .
$$

If $[a]_{p}$ is given to the bit-decomposition protocol as input, it outputs

$$
[a]_{B}=\left([1]_{p},[1]_{p},[1]_{p},[1]_{p},[1]_{p},[0]_{p},[0]_{p},[1]_{p}\right) \text {; }
$$

if $[a]_{p}$ and $m=2$ (or $m=4,8,16,32, \ldots$ ) are given to our Base- $m$ Digit-BitDecomposition protocol as inputs, it will output the same result with the bitdecomposition protocol above; however, when $[a]_{p}$ and $m=10$ are given to our Base-m Digit-Bit-Decomposition protocol, it will output

$\left([2]_{B},[4]_{B},[9]_{B}\right)=\left(\left([0]_{p},[0]_{p},[1]_{p},[0]_{p}\right),\left([0]_{p},[1]_{p},[0]_{p},[0]_{p}\right),\left([1]_{p},[0]_{p},[0]_{p},[1]_{p}\right)\right)$ which is significantly different from the output of bit-decomposition.

We also propose a simplified version of the protocol, called Base-m DigitDecomposition Protocol, which outputs $\left([2]_{p},[4]_{p},[9]_{p}\right)$ when given $[a]_{p}$ and $m=$ 10 as inputs.

Finally, we strongly recommend the interested readers to read [13] which is the full version of this paper. Many of the details are omitted in the present paper due to space constraints.

\section{$1.2 \quad$ Related Work}

The problem of bit-decomposition is a basic problem in MPC and was partially solved by Algesheimer et al. in [1]. However, their solution is not constant-rounds and can only handle values that are noticeably smaller than $p$. Damgård et al. proposed the first constant-rounds (full) solution to the problem of bit-decomposition in [7. This ice-break work is based on linear secret sharing schemes [211]. Independently, Shoenmakers and Tuyls [16] solved the problem 
of bit-decomposition for multiparty computation based on (Paillier) threshold homomorphic cryptosystems [38. Somewhat surprisingly, Nishide and Ohta proposed solutions for comparison, interval test and equality test of shared secrets without relying on bit-decomposition [12. Their techniques are novel, and have enlightened us a lot. Recently, Toft showed a novel technique that can reduce the communication complexity of bit-decomposition to almost linear [18. Although we do not focus on the "almost linear" property of protocols, some techniques proposed in their paper are so inspiring and enlightening to us. In a followup work, Reistad and Toft proposed a linear bit-decomposition protocol [14]. However, the security of their protocol is non-perfect.

As for the problem of modulo reduction (without bit-decomposition), Guajardo et al. proposed a partial solution to this problem in the threshold homomorphic setting [9]. In [6], Catrina et al. dealt with the non-constant-rounds private modulo reduction protocol with the incomplete accuracy and statistical privacy in the setting where shared secrets are represented as fixed-point numbers.

\section{Preliminaries}

In this section we introduce some important notations and some known primitives which will be frequently mentioned in the rest of the paper.

\subsection{Notations and Conventions}

The multiparty computation considered in this paper is based on linear secret sharing schemes, such as Shamir's [15. As mentioned above, we denote the underlying field as $\mathbb{Z}_{p}$ where $p$ is a prime with binary length $l$.

As in previous works, such as [7] and [12, we assume that the underlying secret sharing scheme allows to compute $[a+b \bmod p]_{p}$ from $[a]_{p}$ and $[b]_{p}$ without communication, and that it allows to compute $[a b \bmod p]_{p}$ from (public) $a \in$ $\mathbb{Z}_{p}$ and $[b]_{p}$ without communication. We also assume that the secret sharing scheme allows to compute $[a b \bmod p]_{p}$ from $[a]_{p}$ and $[b]_{p}$ through communication among the parties. We call this procedure the multiplication protocol. Obviously, for multiparty computation, the multiplication protocol is a dominant factor of complexity as it involves communication. So, as in previous works, the round complexity of the protocols is measured by the number of rounds of parallel invocations of the multiplication protocol, and the communication complexity is measured by the number of invocations of the multiplication protocol. For example, if a protocol involves $a$ multiplications in parallel and then another $b$ multiplications in parallel, then we can say that the round complexity is 2 and the communication complexity is $a+b$ multiplications. We have to say that the complexity analysis made in this paper is somewhat rough for we focus mainly on the ideas of the solution, but not on the details of the implementation.

As in [12], when we write $[C]_{p}$, where $C$ is a Boolean test, it means that $C \in\{0,1\}$ and $C=1$ iff $C$ is true. For example, we use $[x \stackrel{?}{<} y]_{p}$ to denote the output of the comparison protocol, i.e. $(x \stackrel{?}{<} y)=1$ iff $x<y$ holds. 
For the base $m \in\{2,3, \ldots, p-1\}$, define $L(m)=\lceil\log m\rceil$. It is easy to see that we should use $L(m)$ bits to represent a base- $m$ digit. For example, when $m=10$, we have $L(m)=\lceil\log 10\rceil=4$, this means we must use 4 bits to represent a base10 digit. Notice that we have $2^{L(m)-1}<m \leq 2^{L(m)}$ and $m=2^{L(m)}$ holds iff $m$ is a power of 2 . Moreover, we have $L(m) \leq l$ as $m \leq p-1$.

Define $l^{(m)}=\left\lceil\log _{m} p\right\rceil$. Obviously, $l^{(m)}$ is the length of $p$ when $p$ is coded base- $m$. Note that $l^{(m)}=\left\lceil\log _{m} p\right\rceil=\left\lceil\frac{\log p}{\log m}\right\rceil=\left\lceil\frac{l}{\log m}\right\rceil \leq l$ as $m \geq 2$.

For any $a \in \mathbb{Z}_{p}$, the secret sharing of $a$ is denoted by $[a]_{p}$. We use $[a]_{B}$ to denote the bitwise sharing of $a$.

We use $[a]_{D}^{m}=\left(\left[a_{l^{(m)}-1}\right]_{p}^{m}, \ldots,\left[a_{1}\right]_{p}^{m},\left[a_{0}\right]_{p}^{m}\right)$ to denote the digit-wise sharing of $a$. For $i \in\left\{0,1, \ldots, l^{(m)}-1\right\},\left[a_{i}\right]_{p}^{m}$ denotes the sharing of the $i^{\prime}$ th base- $m$ digit of $a$ with $0 \leq a_{i} \leq(m-1)$.

The digit-bit-wise sharing of $a$, which is denoted by $[a]_{D, B}^{m}$, is defined as below:

$$
[a]_{D, B}^{m}=\left(\left[a_{l^{(m)}-1}\right]_{B}^{m}, \ldots,\left[a_{1}\right]_{B}^{m},\left[a_{0}\right]_{B}^{m}\right),
$$

in which $\left[a_{i}\right]_{B}^{m}=\left(\left[a_{i}^{L(m)-1}\right]_{p}, \ldots,\left[a_{i}^{1}\right]_{p},\left[a_{i}^{0}\right]_{p}\right)\left(i \in\left\{0,1, \ldots, l^{(m)}-1\right\}\right)$ denotes the bitwise sharing of the $i^{\prime}$ th base- $m$ digit of $a$. Note that $\left[a_{i}\right]_{B}^{m}$ has $L(m)$ bits.

Sometimes, if $m$ can be inferred from the context, we may write $\left[a_{i}\right]_{p}^{m}$ (or $\left.\left[a_{i}\right]_{B}^{m}\right)$ as $\left[a_{i}\right]_{p}\left(\right.$ or $\left.\left[a_{i}\right]_{B}\right)$ for simplicity.

In this paper, we often need to get the digit-wise representation or the digitbit-wise representation of some public value $c$, i.e. $[c]_{D}^{m}$ or $[c]_{D, B}^{m}$. This can be done freely as $c$ is public.

It's easy to see that if we have obtained $[x]_{B}$, then $[x]_{p}$ can be freely obtained by a linear combination. We can think of this as $[x]_{B}$ contains "more information" than $[x]_{p}$. For example, if we get $[a]_{D, B}^{m}=\left(\left[a_{l(m)-1}\right]_{B}^{m}, \ldots,\left[a_{1}\right]_{B}^{m},\left[a_{0}\right]_{B}^{m}\right)$, then $[a]_{D}^{m}=\left(\left[a_{l^{(m)}-1}\right]_{p}^{m}, \ldots,\left[a_{1}\right]_{p}^{m},\left[a_{0}\right]_{p}^{m}\right)$ is implicitly obtained. In protocols that can output both $[x]_{B}$ and $[x]_{p}$, which is often the case in this paper, we always omit $[x]_{p}$ for simplicity.

Given $[c]_{p}$, we need a protocol to reveal $c$, which is denoted by $c \leftarrow \operatorname{reveal}\left([c]_{p}\right)$.

When we write command $C \leftarrow b$ ? $A: B$, where $A, B, C \in \mathbb{Z}_{p}$ and $b \in\{0,1\}$, it means the following:

$$
\text { if } b=1 \text {, then } C \text { is set to } A \text {; otherwise, } C \text { is set to } B \text {. }
$$

We call this command the conditional selection command. When all the variables in this command are public, this "selection" can of course be done. When the variables are shared or even bitwise shared, this can also be done. Specifically, the command

$$
[C]_{p} \leftarrow[b]_{p} ?[A]_{p}:[B]_{p}
$$

can be realized by setting

$$
[C]_{p} \leftarrow[b]_{p}\left([A]_{p}-[B]_{p}\right)+[B]_{p}
$$

which costs 1 round and 1 multiplication; the command

$$
[C]_{B} \leftarrow[b]_{p} ?[A]_{B}:[B]_{B}
$$


can be realized by the following procedure:

For $i=0,1, \ldots, l-1$ in parallel: $\left[C_{i}\right]_{p} \leftarrow[b]_{p}\left(\left[A_{i}\right]_{p}-\left[B_{i}\right]_{p}\right)+\left[B_{i}\right]_{p}$
$[C]_{B} \leftarrow\left(\left[C_{l-1}\right]_{p}, \ldots,\left[C_{1}\right]_{p},\left[C_{0}\right]_{p}\right) \quad \triangleright|A|=|B|=|C|=l$

Note that the above procedure costs 1 round, $l$ invocations of multiplication. Other cases, such as $[C]_{D}^{m} \leftarrow[b]_{p} ?[A]_{D}^{m}:[B]_{D}^{m}$ and $[C]_{D, B}^{m} \leftarrow[b]_{p} ?[A]_{D, B}^{m}$ : $[B]_{D, B}^{m}$ can be realized similarly. We will frequently use this conditional selection command in our protocols.

\subsection{Known Primitives}

We will now simply introduce some existing primitives which are important building blocks of this paper. All these primitives are proposed in [7.

$\Delta$ Random-Bit. The Random-Bit protocol is the most basic primitive which can generate a shared uniformly random bit unknown to all parties. In the linear secret sharing setting, which is the case in this paper, it takes only 2 rounds and 2 multiplications.

$\Delta$ Bitwise-Less Than. Given two bitwise shared inputs $[x]_{B}$ and $[y]_{B}$, the BitwiseLessThan protocol can compute a shared bit $[x \stackrel{?}{<} y]_{p}$. We note that using the method of [18, this protocol can be realized in 6 rounds and $13 l+6 \sqrt{l}$ multiplications. Notice that $13 l+6 \sqrt{l} \leq 14 l$ holds for $l \geq 36$ which is often the case in practice. So, for simplicity, we refer to the complexity of this protocol as 6 rounds and $14 l$ multiplications.

\Bitwise-Addition. Given two bitwise shared inputs, $[x]_{B}$ and $[y]_{B}$, the BitwiseAddition protocol outputs $[x+y]_{B}$. An important point of this protocol is that $d=x+y$ holds over the integers, not (only) mod $p$. This protocol, which costs 15 rounds and $47 l \log l$ multiplications, is the most expensive primitive of the bitdecomposition protocol of [7. We will not use this primitive in this paper, but use Bitwise-Subtraction instead. However, the asymptotic complexity of our BitwiseSubtraction protocol is the same with that of the Bitwise-Addition since they both involve a generic prefix protocol which costs $O(l \log l)$ multiplications. We will introduce our Bitwise-Subtraction protocol later.

\section{A Simple Introduction to Our New Primitives}

In this section, we will simply introduce the new primitives proposed in this paper. We will only describe the inputs and the outputs of the protocols, along with some simple comments. All these new primitives will be described in detail in Section 6.

-Bitwise-Subtraction. The Bitwise-Subtraction protocol accepts two bitwise shared values $[x]_{B}$ and $[y]_{B}$ and outputs $[x-y]_{B}$. This protocol is in fact first proposed in [18] and is re-described (in a widely different form) in this paper. In our protocols, we only need a restricted version (of Bitwise-Subtraction) which requires $x \geq y$. A run of this restricted protocol is denoted by 


$$
[x-y]_{B} \leftarrow \text { Bitwise }- \text { Subtraction } *\left([x]_{B},[y]_{B}\right) .
$$

It costs 15 rounds and $47 l \log l$ multiplications.

-BORROWS. This protocol is used as a sub-protocol in the Bitwise-Subtraction protocol above to compute the borrow bits (as well as in the Bitwise-Subtraction* protocol). Given two bitwise sharings $[x]_{B}$ and $[y]_{B}$, this protocol outputs

$$
\left(\left[b_{l-1}\right]_{p}, \ldots,\left[b_{1}\right]_{p},\left[b_{0}\right]_{p}\right) \leftarrow \operatorname{BORROWS}\left([x]_{B},[y]_{B}\right)
$$

where $\left[b_{i}\right]_{p}$ is the sharing of the borrow bit at bit-position $i \in\{0,1, \ldots, l-1\}$.

-Random-Digit-Bit. Given $m \in\{2,3, \ldots, p-1\}$ as input, the Random-DigitBit protocol outputs

$$
[d]_{B}^{m}=\left(\left[d^{L(m)-1}\right]_{p}, \ldots,\left[d^{1}\right]_{p},\left[d^{0}\right]_{p}\right) \leftarrow \text { Random }-\operatorname{Digit}-\operatorname{Bit}(m)
$$

where $d \in\{0,1, \ldots, m-1\}$ represents a base- $m$ digit. Notice that $[d]_{p}^{m}$ is implicitly obtained. The complexity of this protocol is 8 rounds and $16 L(m)$ multiplications.

-Digit-Bit-wise-LessThan. The Digit-Bit-wise-LessThan protocol accepts two digit-bit-wise shared values $[x]_{D, B}^{m}$ and $[y]_{D, B}^{m}$ and outputs

$$
[x \stackrel{?}{<} y]_{p} \leftarrow \text { Digit }- \text { Bit - wise - LessThan }\left([x]_{D, B}^{m},[y]_{D, B}^{m}\right) .
$$

The complexity of this protocol is 6 rounds and $14 l$ multiplications.

-Random-Solved-Digits-Bits.Using the above two primitives as sub-protocols, we can construct the Random-Solved-Digits-Bits protocol which, when given $m \in$ $\{2,3, \ldots, p-1\}$ as input, outputs a digit-bit-wise shared random value $[r]_{D, B}^{m}$ satisfying $r<p$. We denote a run of this protocol by

$$
[r]_{D, B}^{m} \leftarrow \text { Random - Solved - Digits - Bits }(m) .
$$

This protocol takes 14 rounds and $312 l$ multiplications.

-Digit-Bit-wise-Subtraction. This protocol is a novel generalization to the bitwise subtraction protocol and is very important to this paper. It accepts two digit-bit-wise shared values $[x]_{D, B}^{m}$ and $[y]_{D, B}^{m}$ and outputs $[x-y]_{D, B}^{m}$. Again, in this paper, we need only a restricted version which requires $x \geq y$. A run of this restricted protocol is denoted by

$$
[x-y]_{D, B}^{m} \leftarrow \text { Digit }- \text { Bit - wise }- \text { Subtraction } *\left([x]_{D, B}^{m},[y]_{D, B}^{m}\right) .
$$

This restricted protocol costs 30 rounds and $47 l \log l+47 l \log (L(m))$ multiplications. What's more, if we don't need $[x-y]_{D, B}^{m}$ but (only) need $[x-y]_{D}^{m}$ instead, then this restricted protocol can be (further) simplified. We denote a run of this (further) simplified protocol by

$$
[x-y]_{D}^{m} \leftarrow \text { Digit }- \text { Bit }- \text { wise }- \text { Subtraction }{ }^{*-}\left([x]_{D, B}^{m},[y]_{D, B}^{m}\right) .
$$

The complexity of this protocol goes down to 21 rounds and $16 l+47 l^{(m)} \log \left(l^{(m)}\right)$ multiplications.

With the above primitives, we can construct our Modulo-Reduction protocol and Base-m Digit-Bit-Decomposition protocol, which will be described in detail separately in Section 4 and Section 5 . 


\section{Multiparty Computation for Modulo Reduction without Bit-Decomposition}

In this section, we give out our (public) Modulo-Reduction protocol which is realized without relying on bit-decomposition. This protocol is constant-rounds and involves only $O(l)$ multiplications. Informally speaking, our Modulo-Reduction protocol is essentially the Least Significant Digit Protocol and is a natural generalization to the Least Significant Bit Protocol (i.e. the $L S B$ protocol) in [12. Recall that for an integer $a$, the sharing of the least significant base- $m$ digit of $a$ is denoted by $\left[a_{0}\right]_{p}^{m}$, and the bitwise sharing of the least significant base- $m$ digit of $a$ is denoted by $\left[a_{0}\right]_{B}^{m}$. The protocol is described in detail in Protocol 1.

Protocol 1. The modulo reduction protocol, Modulo-Reduction $(\cdot)$, for computing the residue of a shared integer modulo a public integer.

Input: $[x]_{p}$ with $x \in \mathbb{Z}_{p}$ and $m \in\{2,3, \ldots, p-1\}$.

Output: $[x \bmod m]_{p}$.

Process:

$[r]_{D, B}^{m} \leftarrow$ Random - Solved - Digits - Bits $(m)$

$c \leftarrow$ reveal $\left([x]_{p}+[r]_{D, B}^{m}\right) \quad \triangleright$ Note that $[r]_{D, B}^{m}$ implies $[r]_{p}^{m}$.

$\left[X_{1}\right]_{p}^{m} \leftarrow\left[c_{0}\right]_{p}^{m}-\left[r_{0}\right]_{B}^{m} \quad \triangleright\left[r_{0}\right]_{B}^{m}$ implies $\left[r_{0}\right]_{p}^{m}$.

$\left[X_{2}\right]_{p}^{m} \leftarrow\left[c_{0}\right]_{p}^{m}-\left[r_{0}\right]_{B}^{m}+m$

$[s]_{p} \leftarrow$ Bitwise - LessThan $\left(\left[c_{0}\right]_{B}^{m},\left[r_{0}\right]_{B}^{m}\right)$

$[X]_{p}^{m} \leftarrow[s]_{p} ?\left[X_{2}\right]_{p}^{m}:\left[X_{1}\right]_{p}^{m} \quad \triangleright \mathrm{A}$ conditional selection command.

$c^{\prime} \leftarrow c+p \quad \triangleright$ Addition over the integers.

$\left[X_{1}^{\prime}\right]_{p}^{m} \leftarrow\left[c_{0}^{\prime}\right]_{p}^{m}-\left[r_{0}\right]_{B}^{m}$

$\left[X_{2}^{\prime}\right]_{p}^{m} \leftarrow\left[c_{0}^{\prime}\right]_{p}^{m}-\left[r_{0}\right]_{B}^{m}+m$

$\left[s^{\prime}\right]_{p} \leftarrow$ Bitwise - LessThan $\left(\left[c_{0}^{\prime}\right]_{B}^{m},\left[r_{0}\right]_{B}^{m}\right)$

$\left[X^{\prime}\right]_{p}^{m} \leftarrow\left[s^{\prime}\right]_{p} ?\left[X_{2}^{\prime}\right]_{p}^{m}:\left[X_{1}^{\prime}\right]_{p}^{m}$

$[t]_{p} \leftarrow$ Digit - Bit - wise - LessThan $\left([c]_{D, B}^{m},[r]_{D, B}^{m}\right)$

$[x \bmod m]_{p}=\left[x_{0}\right]_{p}^{m} \leftarrow[t]_{p} ?\left[X^{\prime}\right]_{p}^{m}:[X]_{p}^{m}$

Return $[x \bmod m]_{p}$

Correctness: By simulating a base-m addition process, the protocol extracts $\left[x_{0}\right]_{p}^{m}$ which is just $[x \bmod m]_{p}$. See [13] for the details.

Privacy: The only possible information leakage takes place in line (1.a), where a reveal command is involved. However, the revealed value, i.e. $c$, is uniformly random, so it leaks no information about the secret $x$. So the privacy is guaranteed.

Complexity: Complexity comes mainly from the invocations of sub-protocols. Note that the two invocations of Bitwise-LessThan and the invocation of DigitBit-wise-LessThan can be scheduled in parallel. In all it will cost 22 rounds and

$$
312 l+14 L(m)+1+14 L(m)+1+14 l+1=326 l+28 L(m)+3
$$

multiplications. Recall that $L(m) \leq l$, so the communication complexity is upper bounded by $354 l+3$ multiplications. 
The original modulo reduction problem does not require the sharings of the bits of the residue, i.e. $[x \bmod m]_{B}$. So in the above protocol, $[x \bmod m]_{B}$ is not computed. However, if we want, we can get $[x \bmod m]_{B}$ using an enhanced version of the above Modulo-Reduction protocol. This enhanced protocol will be denoted by Modulo-Reduction ${ }^{+}(\cdot)$. The construction is seen as Protocol 2.

Protocol 2. The enhanced modulo reduction protocol, Modulo-Reduction ${ }^{+}(\cdot)$, for computing the bitwise shared residue of a shared integer modulo a public integer.

Input: $[x]_{p}$ with $x \in \mathbb{Z}_{p}$ and $m \in\{2,3, \ldots, p-1\}$.

Output: $[x \bmod m]_{B}$.

Process:

$[r]_{D, B}^{m} \leftarrow$ Random - Solved - Digits - Bits $(m)$

$c \leftarrow \operatorname{reveal}\left([x]_{p}+[r]_{D, B}^{m}\right)$

$\left[\bar{M}_{1}\right]_{B}^{m} \leftarrow\left[c_{0}\right]_{B}^{m} \quad\left[\bar{S}_{1}\right]_{B}^{m} \leftarrow\left[r_{0}\right]_{B}^{m}$

$\left[\bar{M}_{2}\right]_{B}^{m} \leftarrow\left[c_{0}+m\right]_{B}^{m} \quad\left[\bar{S}_{2}\right]_{B}^{m} \leftarrow\left[r_{0}\right]_{B}^{m} \quad \triangleright$ Addition over the integers.

$[s]_{p} \leftarrow$ Bitwise - LessThan $\left(\left[c_{0}\right]_{B}^{m},\left[r_{0}\right]_{B}^{m}\right)$

$[\bar{M}]_{B}^{m} \leftarrow[s]_{p} ?\left[\bar{M}_{2}\right]_{B}^{m}:\left[\bar{M}_{1}\right]_{B}^{m} \quad \triangleright$ Involving $L(m)$ multiplications.

$[\bar{S}]_{B}^{m} \leftarrow[s]_{p} ?\left[\bar{S}_{2}\right]_{B}^{m}:\left[\bar{S}_{1}\right]_{B}^{m}$

$c^{\prime} \leftarrow c+p$

$\left[\bar{M}_{1}^{\prime}\right]_{B}^{m} \leftarrow\left[c_{0}^{\prime}\right]_{B}^{m} \quad\left[\bar{S}_{1}^{\prime}\right]_{B}^{m} \leftarrow\left[r_{0}\right]_{B}^{m}$

$\left[\bar{M}_{2}^{\prime}\right]_{B}^{m} \leftarrow\left[c_{0}^{\prime}+m\right]_{B}^{m} \quad\left[\bar{S}_{2}^{\prime}\right]_{B}^{m} \leftarrow\left[r_{0}\right]_{B}^{m}$

$\left[s^{\prime}\right]_{p} \leftarrow$ Bitwise - LessThan $\left(\left[c_{0}^{\prime}\right]_{B}^{m},\left[r_{0}\right]_{B}^{m}\right)$

$\left[\bar{M}^{\prime}\right]_{B}^{m} \leftarrow\left[s^{\prime}\right]_{p} ?\left[\bar{M}_{2}^{\prime}\right]_{B}^{m}:\left[\bar{M}_{1}^{\prime}\right]_{B}^{m}$

$\left[\bar{S}^{\prime}\right]_{B}^{m} \leftarrow\left[s^{\prime}\right]_{p} ?\left[\bar{S}_{2}^{\prime}\right]_{B}^{m}:\left[\bar{S}_{1}^{\prime}\right]_{B}^{m}$

$[t]_{p} \leftarrow$ Digit - Bit - wise - LessThan $\left(c,[r]_{D, B}^{m}\right)$

$[M]_{B}^{m} \leftarrow[t]_{p} ?\left[\bar{M}^{\prime}\right]_{B}^{m}:[\bar{M}]_{B}^{m} \quad \triangleright \mathrm{M}$ is the minuend.

$[S]_{B}^{m} \leftarrow[t]_{p} ?\left[\bar{S}^{\prime}\right]_{B}^{m}:[\bar{S}]_{B}^{m} \quad \triangleright \mathrm{S}$ is the subtrahend.

$[x \bmod m]_{B}=\left[x_{0}\right]_{B}^{m} \leftarrow$ Bitwise - Subtraction $*\left([M]_{B}^{m},[S]_{B}^{m}\right)$

Return $[x \bmod m]_{B}$

The correctness and privacy of this protocol can be proved similarly to the Modulo-Reduction protocol above. By carefully selecting the Minuend and the Subtrahend, we can get the expected result by using only one invocation of the Bitwise-Subtraction* protocol. The overall complexity of this protocol is 37 rounds and

$326 l+28 L(m)+47 L(m) \log (L(m))+6 L(m)=326 l+34 L(m)+47 L(m) \log (L(m))$ multiplications.

\section{A Generalization to Bit-Decomposition}

In this section, we will propose our generalization to bit-decomposition, i.e. the Base-m Digit-Bit-Decomposition protocol. The details of this protocol are 
presented in Protocol 3. The main framework of this protocol is similar to the bit-decomposition protocol of [18].

Protocol 3. The Base-m Digit-Bit-Decomposition protocol,

Digit - Bit - Decomposition $(\cdot, m)$, for converting the sharing of secret $x$ into the digit-bit-wise sharing of $x$.

Input: $[x]_{p}$ with $x \in \mathbb{Z}_{p}$ and the base $m \in\{2,3, \ldots, p-1\}$.

Output: $[x]_{D, B}^{m}$

Process:

$[r]_{D, B}^{m} \leftarrow$ Random - Solved - Digits - Bits $(m)$

$c \leftarrow \operatorname{reveal}\left([x]_{p}+[r]_{D, B}^{m}\right)$

$c^{\prime} \leftarrow c+p$

$[t]_{p} \leftarrow$ Digit - Bit - wise - LessThan $\left([c]_{D, B}^{m},[r]_{D, B}^{m}\right)$

$[\tilde{c}]_{D, B}^{m}=[t]_{p} ?\left[c^{\prime}\right]_{D, B}^{m}:[c]_{D, B}^{m} \quad \triangleright$ Note that $\tilde{c}=x+r$

$[x]_{D, B}^{m} \leftarrow$ Digit - Bit - wise - Subtraction* $\left([\tilde{c}]_{D, B}^{m},[r]_{D, B}^{m}\right)$

Return $[x]_{D, B}^{m}$

Correctness is described in detail in 13 . Privacy is straightforward. The overall complexity of this protocol is $14+6+30=50$ rounds and

$312 l+14 l+(47 l \log l+47 l \log (L(m)))=326 l+47 l \log l+47 l \log (L(m))$

multiplications. The communication complexity is upper bounded by $326 l+$ $94 l \log l$ multiplications as $L(m) \leq l$.

If we do not need $[x]_{D, B}^{m}$ but (only) need $[x]_{D}^{m}$ instead, then the above protocol can be simplified. The method is to replace the Digit-Bit-wise-Subtraction* protocol with the Digit-Bit-wise-Subtraction ${ }^{*-}$ protocol. We call this simplified protocol the Base-m Digit-Decomposition Protocol, a run of which is denoted by Digit - Decomposition $(\cdot, m)$. The correctness and privacy of this protocol can be similarly proved. The complexity goes down to $14+6+21=41$ rounds and

$$
312 l+14 l+\left(16 l+47 l^{(m)} \log \left(l^{(m)}\right)\right)=342 l+47 l^{(m)} \log \left(l^{(m)}\right)
$$

multiplications. Recall that $l^{(m)}=\left\lceil\log _{m} p\right\rceil \leq l$, so the communication complexity is upper bounded by $342 l+47 l \log l$ multiplications.

\section{Realizing the Primitives}

In this section, we will describe in detail the (new) primitives which are essential for the protocols of our paper. Informally, most of the protocols in this section are generalized version of the protocols of [7] from base- 2 to base- $m$ for any $m \geq 2$. It will be seen that, when $m$ is a power of 2 , some of our primitives degenerate to the existing primitives in [7. So, in the complexity analysis, we focus on the case where $m$ is not a power of 2 , i.e. $m<2^{L(m)}$.

\subsection{Bitwise-Subtraction}

We describe the Bitwise-Subtraction protocol here. In fact, this protocol is already proposed in [18. They reduced the problem of bitwise-subtraction to 
the Post-fix Comparison problem. Here, we re-consider the problem of bitwisesubtraction and solve it in a (highly) similar manner to the Bitwise-Addition protocol of 7 .

As is mentioned in Section [3, we will first propose a restricted (bitwisesubtraction) protocol, Bitwise-Subtraction*, which requires that the minuend is not less than the subtrahend. We will only use this restricted version in this paper. The general version without the above restriction can be realized with the help of the Bitwise-LessThan protocol. See [13] for the details. Given a BORROWS protocol that can compute the sharings of the borrow bits, the Bitwise-Subtraction* protocol can be realized as in Protocol 4.

Protocol 4. The restricted bitwise-subtraction protocol,

Bitwise - Subtraction*(·), for computing the bitwise sharing of the difference between two bitwise shared values. This protocol requires that the minuend is not less than the subtrahend.

Input: $[x]_{B}=\left(\left[x_{l-1}\right]_{p}, \ldots,\left[x_{1}\right]_{p},\left[x_{0}\right]_{p}\right)$ and $[y]_{B}=\left(\left[y_{l-1}\right]_{p}, \ldots,\left[y_{1}\right]_{p},\left[y_{0}\right]_{p}\right)$ satisfying $x \geq y$.

Output: $[x-y]_{B}=[d]_{B}=\left(\left[d_{l-1}\right]_{p}, \ldots,\left[d_{1}\right]_{p},\left[d_{0}\right]_{p}\right)$.

Process:

$\left(\left[b_{l-1}\right]_{p}, \ldots,\left[b_{1}\right]_{p},\left[b_{0}\right]_{p}\right) \leftarrow \operatorname{BORROWS}\left([x]_{B},[y]_{B}\right)$

$\left[d_{0}\right]_{p} \leftarrow\left[x_{0}\right]_{p}-\left[y_{0}\right]_{p}+2\left[b_{0}\right]_{p}$

For $i=1,2, \ldots, l-1$ in parallel: $\left[d_{i}\right]_{p} \leftarrow\left[x_{i}\right]_{p}-\left[y_{i}\right]_{p}+2\left[b_{i}\right]_{p}-\left[b_{i-1}\right]_{p}$ $[x-y]_{B}=[d]_{B} \leftarrow\left(\left[d_{l-1}\right]_{p}, \ldots,\left[d_{1}\right]_{p},\left[d_{0}\right]_{p}\right)$

Return $[x-y]_{B}$

Note that the output of this protocol, i.e. $[x-y]_{B}$, is of bit length $l$, not $l+1$. This is because $x \geq y$ holds and thus we do not need a sign bit. Correctness and privacy is straightforward. The complexity of this protocol is 15 rounds and $47 l \log l$ multiplications.

\subsection{Computing the Borrow Bits}

We now describe the BORROWS protocol which can compute the sharings of the borrow bits. In fact our BORROWS protocol is highly similar to the CARRIES protocol in 7]. So only the difference is sketched here. As in 7], we use an operator $\circ: \sum \times \sum \rightarrow \sum$, where $\sum=\{S, P, K\}$, which is defined by $S \circ x=S$ for all $x \in \sum, K \circ x=K$ for all $x \in \sum, P \circ x=x$ for all $x \in \sum$. Here, o represents the borrow-propagation operator, whereas in [7] it represents the carry-propagation operator. When computing $[x-y]_{B}$ (where $x \geq y$ holds) with two bitwise shared inputs

$$
[x]_{B}=\left(\left[x_{l-1}\right]_{p}, \ldots,\left[x_{1}\right]_{p},\left[x_{0}\right]_{p}\right) \text { and }[y]_{B}=\left(\left[y_{l-1}\right]_{p}, \ldots,\left[y_{1}\right]_{p},\left[y_{0}\right]_{p}\right),
$$

for bit-position $i \in\{0,1, \ldots, l-1\}$, let $e_{i}=S$ iff a borrow is set at position $i$ (i.e. $\left.x_{i}<y_{i}\right) ; e_{i}=P$ iff a borrow would be propagated at position $i$ (i.e. $x_{i}=y_{i}$ ); $e_{i}=K$ iff a borrow would be killed at position $i$ (i.e. $x_{i}>y_{i}$ ). It can be easily verified that $b_{i}=1$ (i.e. the $i^{\prime} t h$ borrow bit is set, which means the $i^{\prime} t h$ bit needs 
to borrow a "1" from the $(i+1)^{\prime} t h$ bit) iff $e_{i} \circ e_{i-1} \circ \cdots \circ e_{0}=S$. It can be seen that in the case where $\circ$ represents the borrow-propagation operator and in the case where $\circ$ represents the carry-propagation operator, the rules for $\circ$ (i.e. $S \circ x=S, K \circ x=K$ and $P \circ x=x$ for all $x \in \sum$ ) are completely the same. This means that when computing the borrow bits, once all the $e_{i}{ }^{\prime} s$ are obtained, the residue procedure of our BORROWS protocol will be (completely) the same with that of the CARRIES protocol (in [7]). So, the only difference lies in the procedure of computing the $e_{i}{ }^{\prime} s$, which will be sketched below.

As in [7, we represent $S, P$ and $K$ with bit vectors

$$
(1,0,0),(0,1,0),(0,0,1) \in\{0,1\}^{3} .
$$

Then, for every bit-position $i \in\{0,1, \ldots, l-1\},\left[e_{i}\right]_{B}=\left(\left[s_{i}\right]_{p},\left[p_{i}\right]_{p},\left[k_{i}\right]_{p}\right)$ can be obtained as follows: $\left[s_{i}\right]_{p}=\left[y_{i}\right]_{p}-\left[x_{i}\right]_{p}\left[y_{i}\right]_{p} ;\left[p_{i}\right]_{p}=1-\left[x_{i}\right]_{p}-\left[y_{i}\right]_{p}+2\left[x_{i}\right]_{p}\left[y_{i}\right]_{p}$; $\left[k_{i}\right]_{p}=\left[x_{i}\right]_{p}-\left[x_{i}\right]_{p}\left[y_{i}\right]_{p}$, which in fact need only one multiplication (i.e. $\left.\left[x_{i}\right]_{p}\left[y_{i}\right]_{p}\right)$. Correctness follows readily from the above arguments. Privacy is straightforward. The complexity of the protocol is 15 rounds and $47 l \log l$ multiplications.

\subsection{Random-Digit-Bit}

We will now introduce the Random-Digit-Bit protocol for generating a random bitwise shared base- $m$ digit, which is denoted by $d$ here. In fact, $d$ is a random integer satisfying $0 \leq d \leq m-1$. The details are presented in Protocol 5 .

Protocol 5. The Random-Digit-Bit protocol, Random - Digit - Bit (.), for generating the bitwise sharing of a random digit. The digit is base- $m$ for any $m \geq 2$.

Input: The base $m$ satisfying $2 \leq m \leq p-1$.

Output: $[d]_{B}^{m}=\left(\left[d^{L(m)-1}\right]_{p}, \ldots,\left[d^{1}\right]_{p},\left[d^{0}\right]_{p}\right)$ with $0 \leq d \leq m-1$.

Process:

For $i=0,1, \ldots, L(m)-1$ in parallel: $\left[d^{i}\right]_{p} \leftarrow$ Random $-\operatorname{Bit}()$.

$[d]_{B}^{m} \leftarrow\left(\left[d^{L(m)-1}\right]_{p}, \ldots,\left[d^{1}\right]_{p},\left[d^{0}\right]_{p}\right)$

If $m=2^{L(m)}$, then Return $[d]_{B}^{m}$. Otherwise proceed as below.

$[r]_{p} \leftarrow$ Bitwise - LessThan $\left([d]_{B}^{m}, m\right)$

$r \leftarrow \operatorname{reveal}\left([r]_{p}\right)$

If $r=0$, then abort. Otherwise Return $[d]_{B}^{m}$.

See 13 for the correctness. As for the privacy, when this protocol does not abort, the only information leaked is that $d<m$, which is an a priori fact. As for the complexity, when $m$ is not a power of 2 , the total complexity of one run of this protocol is 8 rounds and $16 L(m)$ multiplications. As in [7], using a Chernoff bound, it can be seen that if this protocol has to be repeated in parallel to get a lower abort probability, then the round complexity is still 8 , and the amortized communication complexity goes up to $4 \times 16 L(m)=64 L(m)$ multiplications. 


\subsection{Digit-Bit-Wise-LessThan}

The Digit-Bit-wise-LessThan protocol proposed here is a natural generalization to the Bitwise-LessThan protocol. Recall that when we write $[C]_{p}$, where $C$ is a Boolean test, it means that $C \in\{0,1\}$ and $C=1$ iff $C$ is true. The details of the protocol are presented in Protocol 6.

Protocol 6. The Digit-Bit-wise-LessThan protocol,

Digit-Bit-wise-LessThan $(\cdot)$, for comparing two digit-bit-wise shared values.

Input: Two digit-bit-wise shared values $[x]_{D, B}^{m}=\left(\left[x_{l^{(m)}-1}\right]_{B}^{m}, \ldots,\left[x_{1}\right]_{B}^{m},\left[x_{0}\right]_{B}^{m}\right)$ and $[y]_{D, B}^{m}=\left(\left[y_{l^{(m)}-1}\right]_{B}^{m}, \ldots,\left[y_{1}\right]_{B}^{m},\left[y_{0}\right]_{B}^{m}\right)$.

Output: $[(x \stackrel{?}{<} y)]_{p}$, where $(x \stackrel{?}{<} y)=1$ iff $x<y$ holds.

Process:

$$
\begin{aligned}
& {[X]_{B} \leftarrow }\left(\left[x_{l^{(m)}-1}^{L(m)-1}\right]_{p}, \ldots,\left[x_{l^{(m)}-1}^{1}\right]_{p},\left[x_{l^{(m)}-1}^{0}\right]_{p},\right. \\
& \ldots \\
& \ldots \\
& \ldots \\
& {\left[x_{1}^{L(m)-1}\right]_{p}, \ldots,\left[x_{1}^{1}\right]_{p},\left[x_{1}^{0}\right]_{p}, } \\
& {\left.\left[x_{0}^{L(m)-1}\right]_{p}, \ldots,\left[x_{0}^{1}\right]_{p},\left[x_{0}^{0}\right]_{p}\right) } \\
& {[Y]_{B} \leftarrow }\left(\left[y_{l^{(m)}-1}^{L(m)-1}\right]_{p}, \ldots,\left[y_{l^{(m)}-1}^{1}\right]_{p},\left[y_{l^{(m)}-1}^{0}\right]_{p},\right. \\
& \ldots \\
& \ldots \\
& \ldots \\
& {\left[y_{1}^{L(m)-1}\right]_{p}, \ldots,\left[y_{1}^{1}\right]_{p},\left[y_{1}^{0}\right]_{p}, } \\
& {\left.\left[y_{0}^{L(m)-1}\right]_{p}, \ldots,\left[y_{0}^{1}\right]_{p},\left[y_{0}^{0}\right]_{p}\right) } \\
& {[(x \stackrel{?}{<} y)]_{p}=[(X \stackrel{?}{<} Y)]_{p} \leftarrow \text { Bitwise - LessThan }\left([X]_{B},[Y]_{B}\right) }
\end{aligned}
$$

Return $[(x \stackrel{?}{<} y)]_{p}$

Correctness is presented in 13 . Privacy follows readily from only using private sub-protocols. The complexity of the protocol is 6 rounds and (about) $14 l$ multiplications.

\subsection{Random-Solved-Digits-Bits}

The Random-Solved-Digits-Bits protocol is an important primitive which can generate a digit-bit-wise shared random value unknown to all parties. It is a natural generalization to the Random-Solved-Bits protocol in [7. The details are presented in Protocol 7.

Recall that the bitwise representation of the most significant base- $m$ digit of $p$ is $\left[p_{l^{(m)}-1}\right]_{B}^{m}=\left(p_{l^{(m)}-1}^{L(m)-1}, \ldots, p_{l^{(m)}-1}^{1}, p_{l^{(m)}-1}^{0}\right)$. Suppose $p_{l^{(m)}-1}^{j}(j \in\{0,1, \ldots$, 
$L(m)-1\})$ is the left-most " 1 " in $\left[p_{l^{(m)}-1}\right]_{B}^{m}$. Then, in order to get an acceptable abort probability, the bit-length of the most significant base- $m$ digit of $r$ should be $j+1$ because an acceptable $r$ must be less than $p$. In this protocol, for simplicity, we assume that $p_{l^{(m)}-1}^{L(m)-1}=1$. Under this assumption, we can generate $\left[r_{l^{(m)}-1}\right]_{B}^{m}$ using the Random-Digit-Bit protocol. If $p_{l^{(m)}-1}^{L(m)-1}=0$, then $\left[r_{l^{(m)}-1}\right]_{B}^{m}$ can be generated by using the Random-Bit protocol directly.

Protocol 7. The Random-Solved-Digits-Bits protocol,

Random-Solved-Digits-Bits(·), for jointly generating a digit-bit-wise shared value which is uniformly random from $\mathbb{Z}_{p}$.

Input: $m$, i.e. the expected base of the digits.

Output: $[r]_{D, B}^{m}$, in which $r$ is a uniformly random value satisfying $r<p$.

Process:

For $i=0,1, \ldots, l^{(m)}-1$ in parallel: $\left[r_{i}\right]_{B}^{m} \leftarrow \operatorname{Random}-\operatorname{Digit}-\operatorname{Bit}(m)$.

$[r]_{D, B}^{m} \leftarrow\left(\left[r_{l^{(m)}-1}\right]_{B}^{m}, \ldots,\left[r_{1}\right]_{B}^{m},\left[r_{0}\right]_{B}^{m}\right)$

$[c]_{p} \leftarrow$ Digit - Bit - wise - LessThan $\left([r]_{D, B}^{m},[p]_{D, B}^{m}\right)$

$c \leftarrow \operatorname{reveal}\left([c]_{p}\right)$

If $c=0$, then abort. Otherwise Return $[r]_{D, B}^{m}$.

The correctness and the privacy is straightforward. The amortized complexity of this protocol is $8+6=14$ rounds and $\left(l^{(m)} \cdot 64 L(m)+14 l\right) * 4=312 l$ multiplications.

\subsection{Digit-Bit-Wise-Subtraction}

In this section, we will describe in detail the restricted version, Digit-Bit-wiseSubtraction*, which requires that the minuend is not less than the subtrahend. The general version, which can be realized using the techniques in the BitwiseSubtraction protocol and which is not used in the paper, is omitted for simplicity.

-The Restricted Digit-Bit-Wise-Subtraction. We will now describe in detail the Digit-Bit-wise-Subtraction* protocol. This protocol is novel and is the most important primitive in our Base-m Digit-Bit-Decomposition protocol. The details are presented in Protocol 8.

Protocol 8. The restricted Digit-Bit-wise-Subtraction protocol,

Digit - Bit - wise - Subtraction* $(\cdot)$, for computing the digit-bit-wise sharing of the difference between two digit-bit-wise shared values with the minuend not less than the subtrahend.

Input: $[x]_{D, B}^{m}=\left(\left[x_{l^{(m)}-1}\right]_{B}^{m}, \ldots,\left[x_{1}\right]_{B}^{m},\left[x_{0}\right]_{B}^{m}\right)$ and

$[y]_{D, B}^{m}=\left(\left[y_{l^{(m)}-1}\right]_{B}^{m}, \ldots,\left[y_{1}\right]_{B}^{m},\left[y_{0}\right]_{B}^{m}\right)$ satisfying $x \geq y$.

Output: $[x-y]_{D, B}^{m}=[d]_{D, B}^{m}=\left(\left[d_{l(m)-1}\right]_{B}^{m}, \ldots,\left[d_{1}\right]_{B}^{m},\left[d_{0}\right]_{B}^{m}\right)$. 
Process:

$$
\begin{aligned}
& {[X]_{B} \leftarrow\left(\left[x_{l^{(m)}-1}^{L(m)-1}\right]_{p}, \ldots,\left[x_{l^{(m)}-1}^{1}\right]_{p},\left[x_{l^{(m)}-1}^{0}\right]_{p},\right.} \\
& \text {... } \\
& \text {... } \\
& {\left[x_{1}^{L(m)-1}\right]_{p}, \ldots,\left[x_{1}^{1}\right]_{p},\left[x_{1}^{0}\right]_{p},} \\
& \left.\left[x_{0}^{L(m)-1}\right]_{p}, \ldots,\left[x_{0}^{1}\right]_{p},\left[x_{0}^{0}\right]_{p}\right) \\
& {[Y]_{B} \leftarrow\left(\left[y_{l^{(m)}-1}^{L(m)-1}\right]_{p}, \ldots,\left[y_{l^{(m)}-1}^{1}\right]_{p},\left[y_{l^{(m)}-1}^{0}\right]_{p},\right.} \\
& \text {... } \\
& \text {... } \\
& {\left[y_{1}^{L(m)-1}\right]_{p}, \ldots,\left[y_{1}^{1}\right]_{p},\left[y_{1}^{0}\right]_{p},} \\
& \left.\left[y_{0}^{L(m)-1}\right]_{p}, \ldots,\left[y_{0}^{1}\right]_{p},\left[y_{0}^{0}\right]_{p}\right) \\
& \left(\left[b_{l^{(m)}-1}^{L(m)-1}\right]_{p}, \ldots,\left[b_{l^{(m)}-1}^{1}\right]_{p},\left[b_{l^{(m)}-1}^{0}\right]_{p},\right. \\
& \text {... } \\
& \text {... } \\
& \text {... } \\
& {\left[b_{1}^{L(m)-1}\right]_{p}, \ldots,\left[b_{1}^{1}\right]_{p},\left[b_{1}^{0}\right]_{p},} \\
& \left.\left[b_{0}^{L(m)-1}\right]_{p}, \ldots,\left[b_{0}^{1}\right]_{p},\left[b_{0}^{0}\right]_{p}\right) \leftarrow \operatorname{BORROWS}\left([X]_{B},[Y]_{B}\right) \\
& {\left[t_{0}^{0}\right]_{p}=\left[x_{0}^{0}\right]_{p}-\left[y_{0}^{0}\right]_{p}+2\left[b_{0}^{0}\right]_{p}} \\
& \text { For } j=1, \ldots, L(m)-1 \text {, in parallel: }\left[t_{0}^{j}\right]_{p}=\left[x_{0}^{j}\right]_{p}-\left[y_{0}^{j}\right]_{p}+2\left[b_{0}^{j}\right]_{p}-\left[b_{0}^{j-1}\right]_{p} \text {. }
\end{aligned}
$$

For $i=1, \ldots, l^{(m)}-1 \mathrm{do}$

$$
\left[t_{i}^{0}\right]_{p}=\left[x_{i}^{0}\right]_{p}-\left[y_{i}^{0}\right]_{p}+2\left[b_{i}^{0}\right]_{p}-\left[b_{i-1}^{L(m)-1}\right]_{p}
$$

For $j=1, \ldots, L(m)-1$, in parallel: $\left[t_{i}^{j}\right]_{p}=\left[x_{i}^{j}\right]_{p}-\left[y_{i}^{j}\right]_{p}+2\left[b_{i}^{j}\right]_{p}-\left[b_{i}^{j-1}\right]_{p}$. End for

$$
C \leftarrow 2^{L(m)}-m \quad \triangleright \text { Note that } C \text { is public. }
$$

For $i=0,1, \ldots, l^{(m)}-1 \mathrm{do}$

$$
\left[t_{i}\right]_{B}^{m} \leftarrow\left(\left[t_{i}^{L(m)-1}\right]_{p}, \ldots,\left[t_{i}^{1}\right]_{p},\left[t_{i}^{0}\right]_{p}\right)
$$

If $m<2^{L(m)}$ then $\quad \triangleright$ Recall that $m<2^{L(m)}$ means $m$ is not a power of 2 .

$$
\left[d_{i}\right]_{B}^{m} \leftarrow \text { Bitwise - Subtraction* }\left(\left[t_{i}\right]_{B}^{m},\left(\left[b_{i}^{L(m)-1}\right]_{p} ? C: 0\right)\right)
$$

Else

End if

$$
\left[d_{i}\right]_{B}^{m} \leftarrow\left[t_{i}\right]_{B}^{m}
$$

End for

$$
\begin{aligned}
& {[x-y]_{D, B}^{m}=[d]_{D, B}^{m} \leftarrow\left(\left[d_{l^{(m)}-1}\right]_{B}^{m}, \ldots,\left[d_{1}\right]_{B}^{m},\left[d_{0}\right]_{B}^{m}\right)} \\
& \text { Return }[x-y]_{D, B}^{m}
\end{aligned}
$$

Correctness is described in detail in [13. Privacy follows readily from the fact that we only call private sub-protocols. The complexity of this protocol 
is 30 rounds and $47 l \log l+47 l \log (L(m))$ multiplications. The communication complexity is upper bounded by $94 l \log l$ multiplications since $L(m) \leq l$.

-A Simplified Version. If we do not need $[x-y]_{D, B}^{m}$ but (only) need $[x-y]_{D}^{m}$ instead, a simplified version of the above protocol, Digit-Bit-wise-Subtraction ${ }^{*-}$, can be obtained by simply replacing all the statements after statement (8.a) with the following.

\section{$\left[d_{0}\right]_{p}^{m}=\left[x_{0}\right]_{p}^{m}-\left[y_{0}\right]_{p}^{m}+m\left[b_{0}^{L(m)-1}\right]_{p}$}

For $i=1, \ldots, l^{(m)}-1$ in parallel: $\left[d_{i}\right]_{p}^{m}=\left[x_{i}\right]_{p}^{m}-\left[y_{i}\right]_{p}^{m}+m\left[b_{i}^{L(m)-1}\right]_{p}-\left[b_{i-1}^{L(m)-1}\right]_{p}$ $[x-y]_{D}^{m}=[d]_{D}^{m} \leftarrow\left(\left[d_{l(m)-1}\right]_{p}^{m}, \ldots,\left[d_{1}\right]_{p}^{m},\left[d_{0}\right]_{p}^{m}\right)$

Return $[x-y]_{D}^{m}$

Note that the above process is free. Correctness and privacy is straightforward. The complexity of this protocol goes down to 15 rounds and $47 l \log l$ multiplications as the expensive Bitwise-Subtraction* protocol is omitted.

If this (simplified) protocol is constructed from scratch, then, for relatively large $m$, the borrow bits for every digit-position, i.e. $\left[b_{i}^{L(m)-1}\right]_{p}$ for $i \in\{0,1, \ldots$, $\left.l^{(m)}-1\right\}$, can be obtained with a lower cost. For every digit-position $i \in$ $\left\{0,1, \ldots, l^{(m)}-1\right\}, e_{i} \in\{S, P, K\}$ can be obtained by calling the linear primitive Bitwise-LessThan. Specifically, we have

$e_{i}=S \Leftrightarrow\left[x_{i}\right]_{B}^{m}<\left[y_{i}\right]_{B}^{m} ; \quad e_{i}=P \Leftrightarrow\left[x_{i}\right]_{B}^{m}=\left[y_{i}\right]_{B}^{m} ; \quad e_{i}=K \Leftrightarrow\left[x_{i}\right]_{B}^{m}>\left[y_{i}\right]_{B}^{m}$.

So, using the Bitwise-Less Than protocol in both ways, which costs $l+\sqrt{l}$ more multiplications and no more rounds than one single invocation [18, we can get all the $e_{i}{ }^{\prime} s$. Then as in the BORROWS protocol (or the CARRIES protocol), the target borrow bits (for every digit-position) can be obtained by using a generic prefix protocol which costs 15 rounds and $47 l^{(m)} \log l^{(m)}$ multiplications. So the Digit-Bit-wise-Subtraction $^{*-}$ protocol can be realized in $6+15=21$ rounds and (less than) $16 l+47 l^{(m)} \log \left(l^{(m)}\right)$ multiplications. Recall that $l^{(m)}=\left\lceil\log _{m} p\right\rceil$. Then for relatively large $m$, e.g. $m \approx p^{\frac{1}{10}}$ where $l^{(m)}=10$, the communication complexity may be very low.

\section{Comments}

As in [12, although we describe all our protocols in the secret sharing setting, our techniques are also applicable to the threshold homomorphic setting. All the protocols in our paper can be similarly realized in this setting. However, some of the protocols in this setting may be less efficient than their counterpart in the secret sharing setting because the Random-Bit protocol, which is a basic building block, is more expensive in the threshold homomorphic setting.

It is easy to see that using our Base-m Digit-Decomposition protocol which extracts all the base- $m$ digits of the shared input, we can also solve the modulo reduction problem (which requires only the least significant base- $m$ digit). However, our Modulo-Reduction protocol is meaningful because it achieves linear communication complexity and thus is much more efficient. 
Obviously, we can say that the bit-decomposition protocol (of [7]) is a special case of our Base-m Digit-Bit-Decomposition protocol when $m$ is a power of 2 . In fact, we can also view the bit-decomposition protocol as a special case of our enhanced Modulo-Reduction protocol when the modulus $m$ is just $p$, i.e. we have

$$
[x]_{B}=\text { Bit }- \text { Decomposition }\left([x]_{p}\right)=\text { Modulo }- \text { Reduction }^{+}\left([x]_{p}, p\right)
$$

for any $x \in \mathbb{Z}_{p}$. Our enhanced Modulo-Reduction protocol can handle not only the special case where $m=p$ but also the general case where $m \in\{2,3, \ldots, p-1\}$, so it can also be viewed as a generalization to bit-decomposition.

We note that, in [18, a novel technique is proposed which can reduce the communication complexity of the bit-decomposition protocol to almost linear. We argue that their technique can also be used in our Base-m Digit-Bit-Decomposition protocol (as well as our Base-m Digit-Decomposition protocol) to reduce the (communication) complexity to almost linear, because their technique is in fact applicable to any PreFix-o (or PostFix-o) protocol (which is a dominant factor of the communication complexity) assuming a linear protocol for computing the UnboundedFanIn - o exists, which is just the case in our protocols.

\section{Applications and Future Work}

In [13, we will show some applications of our new protocols, such as efficient Integer Division protocol, Divisibility Test protocol, Conversion of Integer Representation between Number Systems, etc.

Although we are successful in providing an (efficient) solution to the public modulo reduction problem, we fail in solving the private modulo reduction problem where the modulus is (also) secret shared. The absence of the knowledge of the exact value of $m$ makes our techniques useless. We leave it an open problem to construct efficient protocols for private modulo reduction without relying on bit-decomposition.

Acknowledgments. We would like to thank the anonymous reviewers for their careful work and helpful comments.

\section{References}

1. Algesheimer, J., Camenisch, J.L., Shoup, V.: Efficient Computation Modulo A Shared Secret with Application to the Generation of Shared Safe-Prime Products. In: Yung, M. (ed.) CRYPTO 2002. LNCS, vol. 2442, pp. 417-432. Springer, Heidelberg (2002)

2. Ben-Or, M., Goldwasser, S., Wigderson, A.: Completeness Theorems for Noncryptographic Fault-Tolerant Distributed Computations. In: 20th Annual ACM Symposium on Theory of Computing, pp. 1-10. ACM Press, New York (1988)

3. Cramer, R., Damgård, I.B., Nielsen, J.B.: Multiparty Computation from Threshold Homomorphic Encryption. In: Pfitzmann, B. (ed.) EUROCRYPT 2001. LNCS, vol. 2045, pp. 280-300. Springer, Heidelberg (2001)

4. Chandra, A.K., Fortune, S., Lipton, R.J.: Lower Bounds for Constant Depth Circuits for Prefix Problems. In: Díaz, J. (ed.) ICALP 1983. LNCS, vol. 154, pp. 109-117. Springer, Heidelberg (1983) 
5. Chandra, A.K., Fortune, S., Lipton, R.J.: Unbounded Fan-In Circuits and Associative Functions. In: 15th Annual ACM Symposium on Theory of Computing, pp. 52-60. ACM Press, New York (1983)

6. Catrina, O., Saxena, A.: Secure Computation with Fixed-Point Numbers. In: Sion, R. (ed.) FC 2010. LNCS, vol. 6052, pp. 35-50. Springer, Heidelberg (2010)

7. Damgård, I.B., Fitzi, M., Kiltz, E., Nielsen, J.B., Toft, T.: Unconditionally Secure Constant-Rounds Multi-Party Computation for Equality, Comparison, Bits and Exponentiation. In: Halevi, S., Rabin, T. (eds.) TCC 2006. LNCS, vol. 3876, pp. 285-304. Springer, Heidelberg (2006)

8. Damgård, I.B., Nielsen, J.B.: Universally Composable Efficient Multiparty Computation from Threshold Homomorphic Encryption. In: Boneh, D. (ed.) CRYPTO 2003. LNCS, vol. 2729, pp. 247-264. Springer, Heidelberg (2003)

9. Guajardo, J., Mennink, B., Schoenmakers, B.: Modulo Reduction for Paillier Encryptions and Application to Secure Statistical Analysis. In: Sion, R. (ed.) FC 2010. LNCS, vol. 6052, pp. 375-382. Springer, Heidelberg (2010)

10. Goldreich, O., Micali, S., Wigderson, A.: How to Play Any Mental Game or A Complete Theorem for Protocols with Honest Majority. In: 19th Annual ACM Symposium on Theory of Computing, pp. 218-229. ACM Press, New York (1987)

11. Gennaro, R., Rabin, M.O., Rabin, T.: Simplified Vss and Fast-Track Multiparty Computations with Applications to Threshold Cryptography. In: 17th ACM Symposium on Principles of Distributed Computing, pp. 101-110. ACM Press, New York (1998)

12. Nishide, T., Ohta, K.: Multiparty Computation for Interval, Equality, and Comparison without Bit-Decomposition Protocol. In: Okamoto, T., Wang, X. (eds.) PKC 2007. LNCS, vol. 4450, pp. 343-360. Springer, Heidelberg (2007)

13. Ning, C., Xu, Q.: Multiparty Computation for Modulo Reduction without BitDecomposition and A Generalization to Bit-Decomposition. Cryptology ePrint Archive, Report 2010/266, http://eprint.iacr.org/2010/266

14. Reistad, T., Toft, T.: Linear, Constant-Rounds Bit-Decomposition. In: Lee, D., Hong, S. (eds.) ICISC 2009. LNCS, vol. 5984, pp. 245-257. Springer, Heidelberg (2010)

15. Shamir, A.: How to Share A Secret. Communications of the ACM 22(11), 612-613 (1979)

16. Schoenmakers, B., Tuyls, P.: Efficient Binary Conversion for Paillier Encrypted Values. In: Vaudenay, S. (ed.) EUROCRYPT 2006. LNCS, vol. 4004, pp. 522-537. Springer, Heidelberg (2006)

17. Toft, T.: Primitives and Applications for Multi-party Computation. PhD thesis, University of Aarhus (2007), http://www.daimi.au.dk/ ttoft/publications/dissertation.pdf

18. Toft, T.: Constant-Rounds, Almost-Linear Bit-Decomposition of Secret Shared Values. In: Fischlin, M. (ed.) CT-RSA 2009. LNCS, vol. 5473, pp. 357-371. Springer, Heidelberg (2009) 